\title{
Model-Optimized Dispatch for Closed-Loop Power Control of Waked Wind Farms
}

\author{
Kazda, Jonas; Cutululis, Nicolaos Antonio
}

Published in:

I E E E Transactions on Control Systems Technology

Link to article, DOI:

10.1109/TCST.2019.2923779

Publication date:

2020

Document Version

Peer reviewed version

Link back to DTU Orbit

Citation (APA):

Kazda, J., \& Cutululis, N. A. (2020). Model-Optimized Dispatch for Closed-Loop Power Control of Waked Wind Farms. I E E E Transactions on Control Systems Technology, 28(5), 2029-2036.

https://doi.org/10.1109/TCST.2019.2923779

\section{General rights}

Copyright and moral rights for the publications made accessible in the public portal are retained by the authors and/or other copyright owners and it is a condition of accessing publications that users recognise and abide by the legal requirements associated with these rights.

- Users may download and print one copy of any publication from the public portal for the purpose of private study or research.

- You may not further distribute the material or use it for any profit-making activity or commercial gain

- You may freely distribute the URL identifying the publication in the public portal

If you believe that this document breaches copyright please contact us providing details, and we will remove access to the work immediately and investigate your claim 


\title{
Model-Optimized Dispatch for Closed-Loop Power Control of Waked Wind Farms
}

\author{
Jonas Kazda ${ }^{\circledR}$ and Nicolaos A. Cutululis ${ }^{(\mathbb{D}}$, Senior Member, IEEE
}

\begin{abstract}
At present, wind farms control their power production by using a closed-loop feedback control approach, which distributes the total power to the wind turbines. However, the total power is distributed according to the turbines' available power only. The use of model-predictive control allows considering multiple objectives, nonetheless, since it is open-loop, it can result in poor tracking of the total power reference. This work is the first to combine the standard, closed-loop feedback controller with model-predictive optimization (MPO) in order to yield the benefits of both approaches. As such, we developed an optimization-based dispatch function employed in a closed-loop feedback controller. The dispatch function uses model-predictive, multi-objective optimization to determine the distribution of the total power to the wind turbines. The model employed in the developed dispatch function is the Dynamic Flow Predictor, which uses Kalman-filter-driven feedback to correct the wind farm flow model dynamically. The developed optimization-based dispatch function is compared to dispatch functions commonly employed in present wind farms in a secondary regulation scenario in dynamic simulation. The comparison is carried out on an 80-turbine, large-scale wind farm. The newly developed, optimization-based dispatch function yields a reduction of the mean error and the normalized root-mean-square (NRMS) error by $43 \%$ and $36 \%$ with respect to the best-performing, commonly used dispatch function. Furthermore, for the large-scale wind farm, the duration of the MPO is only $0.21 \mathrm{~s}$, which is two orders of magnitude faster than comparable approaches in the literature.
\end{abstract}

Index Terms-Available power, closed-loop feedback, computational cost, dispatch function, model predictive, power control, wakes, wind energy.

\section{INTRODUCTION}

$\mathbf{T}$ $\mathrm{HE}$ wind energy market has been growing rapidly at a rate of $16 \%$ throughout the past decade reaching $539123 \mathrm{MW}$ of global, installed capacity in 2017 [1]. The increasing share of renewable power generation sources in the electricity market is replacing conventional generation sources. This change is putting pressure on the power system, as conventional generators are used for providing grid balancing services, historically. However, wind power plants could substitute the existing conventional generation sources in providing such grid services [2].

Manuscript received December 3, 2018; accepted June 4, 2019. Manuscript received in final form June 14, 2019. This work was supported by Energinet.dk through the Public Service Obligation Scheme (ForskEL 12396). Recommended by Associate Editor A. Chakrabortty. (Corresponding author: Jonas Kazda.)

The authors are with DTU Wind Energy, Technical University of Denmark, 4000 Roskilde, Denmark (e-mail: kazd@dtu.dk; niac@dtu.dk).

Color versions of one or more of the figures in this paper are available online at http://ieeexplore.ieee.org.

Digital Object Identifier 10.1109/TCST.2019.2923779
To provide grid balancing services and as such to follow a reference for the total power of the wind farm, power controllers of wind farms typically use closed-loop feedback control. The quality of the balancing service and as such the reference tracking performance of the feedback controller depends on its ability to handle the variable, available power of wind turbines. For instance, in case the feedback controller requests a larger power output from a turbine than its available power, a steady-state error results in the turbine's power output. Such error on the turbine level can translate into a steady-state error in the farm power output, and thus, a worse quality of the ancillary service. At present, the power requested from a wind turbine is set by the dispatch function of the power controller of the wind farm. The simplest dispatch function is to distribute the total power to the wind turbines using a static distribution. Due to the variability of the available power of wind turbines, this approach can result in insufficiently accurate tracking of the total power reference. In [3], gain scheduling is proposed to mitigate the effect. Another approach is to distribute the total power to the wind turbines in proportion to the turbines' available power [4]. Albeit the approach improves the handling of the variable, available power of wind turbines compared to the static dispatch, it does not allow for the consideration of more objectives of the operation.

An alternative approach, which is investigated in the literature on power control, is model-predictive control [5]-[7]. The approach has three major advantages. First, it allows optimizing the operation according to multiple objectives. As a result, the approach enables to not only follow a reference for the total power of the wind farm but also to consider other objectives such as the reduction of fatigue loads of wind turbines [5], [6]. Second, the use of models in control can result in more informed control decisions, and hence potentially in improved performance. Typically employed models are dynamic models of wind farm operation [7]-[9] and models of turbine mechanical loads [5], [6]. Third, model-predictive control allows deciding on the present control action considering the potential, future trajectories of operation. In [7] it is shown that accounting for the future dynamics of wind farm flow improves the control performance. Despite these three advantages, there are two impediments to model-predictive control of wind farms. First, the accuracy of tracking a demanding reference for the total power of a waked wind farm is likely to be low, because model-predictive control is open-loop [10]. Second, modeling the dynamics of flow in a large-scale wind farm is computationally challenging with many of the current approaches. Amongst these, engineering 
models typically result in a faster computational speed. Using engineering models in [7] and [11] resulted in a duration of the optimization in the order of minutes. Although remarkably fast, such duration still results in a delay in introducing the optimized control actions to the wind turbines.

To address these challenges, this work introduces a modelpredictive, closed-loop feedback controller including a computationally fast model. The controller is a synthesis of the investigated approaches for power control in the literature, that is model-predictive control and closed-loop feedback control. The outer control loop is the closed-loop feedback controller that is commonly employed in present wind farms as introduced above. It ensures the accurate tracking of the reference for the total power of the wind farm, allowing it to operate in a mode that ensures available active power reserve to be used in ancillary services. In order to provide the advantages of model-predictive control described above, model-predictive optimization (MPO) is used in the dispatch function of the closed-loop feedback controller. To reduce the computation time of the MPO, a linear model is used, the Dynamic Flow Predictor [8].

The structure of the article is as follows. In Section II, the methodology is detailed. Section III presents tests of the optimization-based dispatch function on a large-scale wind farm. This brief concludes with a summary of the key findings in Section IV.

\section{Methods}

First, the closed-loop feedback controller is introduced; second, the static and proportional dispatch functions are described for reference; and finally, the MPO-based dispatch function is presented.

\section{A. Closed-Loop Feedback Controller}

Fig. 1 shows the system structure of the closed-loop, feedback wind farm controller. The wind farm controller consists of a proportional-integral (PI) controller and a dispatch function, which is used to distribute the total power demanded by the PI-controller to the wind turbines.

1) PI-Controller: The aim of the PI-controller is to track the total power reference signal. The transfer function $C(s)$ of the PI-controller is defined as

$$
C(s)=k_{p}\left(1+\frac{1}{s T_{i}}\right)
$$

where $k_{p}$ is the proportional gain and $T_{i}$ is the time constant of the integrator. An Antiwindup-Reset setup is used to limit the integrator with the upper limit set to the wind farm available power.

2) Total Power Dispatch: The output of the PI-controller is the demanded total wind farm power, which is distributed to the wind turbines in the wind farm using the dispatch function. The approach used in the dispatch function for the distribution of the demanded total power $P_{\mathrm{dem}}$,tot depends on the objectives of the wind farm operator. Generally, the demanded total power is distributed to the wind turbines as

$$
P_{\text {set }, j}=f_{j} P_{\text {dem,tot }}
$$

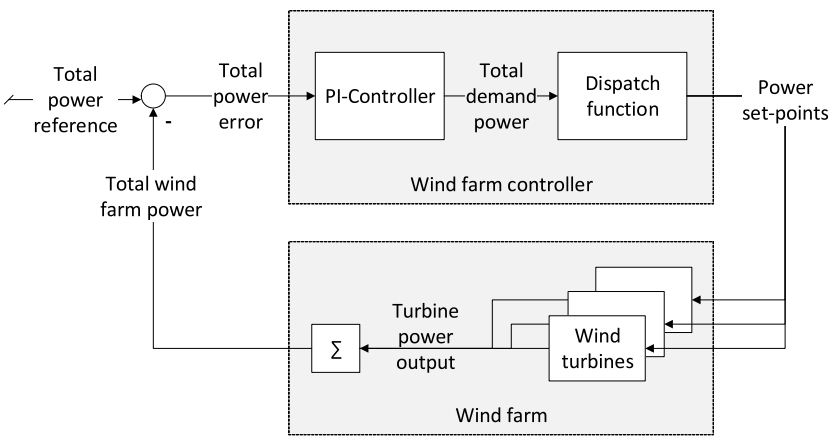

Fig. 1. System structure of closed-loop power controller for wind farms, comprising PI-controller and dispatch function.

where $f_{j}$ is the fraction of the total demanded wind farm power dispatched to turbine $j$ and $P_{\text {set }, j}$ is the power setpoint of turbine $j . f_{j}$ is subject to

$$
1=\sum_{j=1}^{J} f_{j}
$$

where $J$ is the number of wind turbines in the wind farm. Three different dispatch functions are considered in this work, the newly developed MPO-based dispatch, and, for reference, the static dispatch and the proportional dispatch. More details on these are discussed in Sections II-B and II-C.

3) Controller Tuning: The tuning of the feedback controller is conducted in three steps: 1) system identification; 2) system analysis; and 3) system control.

a) System identification: The transfer function from the power set-points of the turbines to the total power output of the wind farm can be described as

$$
P_{\text {tot }}(s)=\sum_{j=1}^{J} G_{j}(s) P_{\text {set }, \mathrm{j}}(s)
$$

where $P_{\text {set, }, j}(s)$ and $P_{\text {tot }}(s)$ are the Laplace-transformed signals of turbine power setpoint and of the total power output of the wind farm, respectively. $G_{j}(s)$ is the transfer function, which models the dynamics between turbine power setpoint and turbine power output, i.e., the turbine operation. The transfer function $G(s)$ was identified from the frequency response of the simulated wind turbine to be well represented as a second-order system

$$
G(s)=\frac{k_{G} \omega_{0}^{2}}{s^{2}+2 \delta \omega_{0} s+\omega_{0}^{2}}
$$

where $\omega_{0}$ is the bandwidth of the closed-loop turbine control system, $\delta$ is the damping factor, and $k_{G}$ is the steady-state gain of the system. For the conditions used in the case study of this work (see Section III-A2), $\omega_{0}$ and $\delta$ are identified to be $18.3 \mathrm{rad} / \mathrm{s}$ and 1.05 , respectively. When the turbine power setpoint is less than or equal to the turbine's available power, the steady-state gain $k_{G}$ is 1 . However, a power setpoint larger than the available power of a turbine results in a steady-state gain below 1. As shown in [3], the result is a reduced speed of the closed-loop wind farm system and potentially a steady-state error from the total power reference. 
Therefore, an objective of the dispatch function can be to ensure that the turbine power set-points are not higher than the turbines' available power.

When the power setpoints of all turbines are less or equal to the available power of these turbines, $G_{j}(s)$ can be assumed to be the same for all turbines. Furthermore, using (2) and (4) can be written as

$$
P_{\mathrm{tot}}(s)=G(s) P_{\mathrm{dem}, \mathrm{tot}}(s) \sum_{j=1}^{J} f_{j} .
$$

Using (3) and (6), the system controlled by the PI-controller is thus modeled as

$$
P_{\text {tot }}(s)=G(s) P_{\text {dem,tot }}(s) \text {. }
$$

The PI-controller is, therefore, designed for the system transfer function $G(s)$.

b) System analysis: The system $G(s)$ has two poles with real part smaller than zero and is thus asymptotically stable, as expected. In this simulation framework, the wind farm controller obtains measurements from the wind turbines at a frequency of $1 \mathrm{~Hz}$. Due to the slow sampling frequency, a discrete time controller synthesis is required.

c) System control: The system $G(s)$ and the PI-controller $C(s)$ are, thus, converted to discrete time. $G(s)$ is converted using a zero-order hold and $C(s)$ is converted using the Tustin method. The resulting controller transfer function $C(z)$ is

$$
C(z)=k_{p}\left(1+\frac{T_{s}}{2 T_{i}} \frac{z+1}{z-1}\right)
$$

where $T_{S}$ is the sampling time. The poles and zeros of the closed-loop system are tuned with the time-domain objective to achieve a fast rise time and no overshoot. The Nyquist diagram shows that the open-loop transfer function $L(z)=$ $G(z) C(z)$ does not enclose the critical point -1 . Since the poles of the open-loop transfer function are located at $\pi(L(z))=\{1 ; 1.8 e-6 ; 8.4 e-12\}$, the closed-loop system is stable according to the Nyquist criterion. Furthermore, the large minimum distance of 0.7 from the critical point -1 shows the robustness of the closed-loop system.

\section{B. Static and Proportional Dispatch}

The developed MPO-based dispatch is benchmarked with the two standard dispatch approaches, that is the static dispatch and the proportional dispatch. The static dispatch distributes the demanded total power to the turbines according to a constant distribution. The proportional dispatch [4] distributes the total demanded power according to the available aerodynamic power at wind turbines.

\section{Model-Predictive Optimized Dispatch}

The advantages of using a dispatch function based on MPO are, as described in Section I, predictive control and the use of multiple objectives and models of operation. This work aims to introduce the MPO-based dispatch and, therefore, only focuses on the most important objective, i.e., reference tracking used for wind farm ancillary services.
1) Objective Function: The objectives of the MPO cost function $\mathcal{J}$ are to: 1) follow the total power reference and 2) to penalize the variation of the turbine power. The cost function is, thus, defined as

$$
\begin{aligned}
\mathcal{J}(\underline{v})=\sum_{k=0}^{N}\{ & \left(P_{\mathrm{MPO}, \text { tot }}[n+k]-P_{\mathrm{ref}}[n+k]\right)^{2}+\sum_{j=1}^{J} \rho_{\text {rate }} \\
& \left.\times\left(P_{\mathrm{MPO}, j}[n+k]-P_{\mathrm{MPO}, j}[n-1+k]\right)^{2}\right\}
\end{aligned}
$$

where $P_{\mathrm{MPO}, \text { tot }}[n]$ is the sum power output of all turbines in the wind farm at time step $n$ and $P_{\text {ref }}[n]$ is the total power reference. $\rho_{\text {rate }}$ is the weighting factor for the rate of turbine power change. It was tuned by simulation prior to the case study with the objective to improve the accuracy in tracking the reference for the total power of the wind farm. $P_{\mathrm{MPO}, j}[n]$ is the power setpoint and equally the power output of turbine $j$ at time step $n$. The decision variable $\underline{v} \in \mathbb{R}^{N * J}$ is the power setpoints of all turbines over the control horizon $N$. Generally, in this work, the underline denotes that the quantity or matrix spans over the entire prediction horizon.

The power setpoints derived in the MPO are used in the dispatch function of the closed-loop feedback controller as

$$
P_{\mathrm{set}, j}=\frac{P_{\mathrm{MPO}, j}}{\sum_{j=1}^{J} P_{\mathrm{MPO}, j}} P_{\mathrm{dem}, \text { tot }} .
$$

2) Constraints: The turbine power setpoints $\underline{v}$ are constrained by the availability of aerodynamic power at the wind turbines. Such constraint is of importance since requesting more power than available from a turbine can result in a steady state error from the reference for the total power of the wind farm. In this work, the turbines' available power is estimated using a newly developed, dynamic, linear model. The model predicts the turbine available power based on wind farm aerodynamics and turbine power setpoints. The aerodynamic interaction of wind turbines in the wind farm is modeled using the Dynamic Flow Predictor [8]. The future aerodynamic power $\underline{P}_{\text {avail }}$ of all wind turbines is calculated as

$$
\underline{P}_{\text {avail }}(\underline{v})=\underline{P}_{\text {avail }, 0}+\underline{\mathbf{B}}_{\mathrm{dP}}\left(\underline{u}(\underline{v})-\underline{u}_{0}\right) .
$$

The matrix $\underline{\mathbf{B}}_{\mathrm{dP}}$ is a diagonal matrix defined as

$$
\underline{\mathbf{B}}_{\mathrm{dP}}=\operatorname{diag}\left(\mathbf{B}_{\mathrm{dP}}[\mathrm{n}], \mathbf{B}_{\mathrm{dP}}[\mathrm{n}+1], \ldots, \mathbf{B}_{\mathrm{dP}}[\mathrm{n}+\mathrm{N}]\right)
$$

where $\mathbf{B}_{\mathrm{dP}}$ is a diagonal matrix defined as

$$
\mathbf{B}_{\mathrm{dP}}=\operatorname{diag}\left(\left.\frac{\partial \mathrm{P}_{1}}{\partial \mathrm{u}_{1}}\right|_{\mathrm{x}_{0}},\left.\frac{\partial \mathrm{P}_{2}}{\partial \mathrm{u}_{2}}\right|_{\mathrm{x}_{0}}, \ldots,\left.\frac{\partial \mathrm{P}_{\mathrm{J}}}{\partial \mathrm{u}_{\mathrm{J}}}\right|_{\mathrm{x}_{0}}\right)
$$

where $\left.(\partial P / \partial u)\right|_{x_{0}}$ is the derivative of turbine power $P$ to wind speed $u$ at the operation point of that time step. The derivative of wind turbine power to wind speed is calculated using the turbine's power curve. The operation point of a wind turbine is estimated using the Dynamic Flow Predictor for each prediction step.

$\underline{P}_{\text {avail }, 0}$ is the baseline, available power of the wind turbines over the prediction horizon. It is calculated assuming that 
the turbines continue operating at the same current power setpoints, that is $\underline{v}_{0} \cdot \underline{P}_{\mathrm{avail}, 0}$ is calculated as

$$
\underline{P}_{\text {avail }, 0}=\frac{1}{2} \rho A c_{P, \max } \underline{u}_{0}\left(\underline{v}_{0}\right)^{3}
$$

where $\rho$ is the density of the air, $A$ is the rotor area, and $c_{P, \max }$ is the maximum power coefficient of a turbine. The wind speed predictions $\underline{u}_{0}$ are obtained using the Dynamic Flow Predictor as

$$
\begin{aligned}
\underline{u}_{0}\left(\underline{v}_{0}\right) & =\underline{\mathbf{C}}_{\mathbf{u}} \underline{x}\left(\underline{v}_{0}\right) \\
& =\underline{\mathbf{C}}_{\mathbf{u}}\left(\underline{\mathbf{A}}_{\vec{x}}+\underline{\mathbf{B}}_{0}\right)
\end{aligned}
$$

where matrix $\underline{\mathbf{C}}_{\mathbf{u}}$ is used to obtain the wind speed estimate at wind turbines from the state vector $\underline{x}$ of the Dynamic Flow Predictor state space system. The matrices $\underline{\mathbf{A}}$ and $\underline{\mathbf{B}}$ are defined based on the principles of model predictive control [12] as

$$
\begin{aligned}
\underline{\mathbf{A}} & =\left[\begin{array}{c}
\mathbf{I} \\
\mathbf{A} \\
\mathbf{A}^{2} \\
\ldots \\
\mathbf{A}^{N}
\end{array}\right] \\
\underline{\mathbf{B}} & =\left[\begin{array}{cccc}
\mathbf{0} & \mathbf{0} & \ldots & \mathbf{0} \\
\mathbf{B} & \mathbf{0} & \ldots & \mathbf{0} \\
\mathbf{A B} & \mathbf{B} & \ldots & \mathbf{0} \\
\vdots & \vdots & \ddots & \vdots \\
\mathbf{A}^{N-1} \mathbf{B} & \mathbf{A}^{N-2} \mathbf{B} & \ldots & \mathbf{0}
\end{array}\right]
\end{aligned}
$$

where $\mathbf{A}$ and $\mathbf{B}$ are the matrices of the Dynamic Flow Predictor state space system, as shown in (19). The wind speed predictions $\underline{u}(\underline{v})$ are obtained equivalently to (15).

Finally, using the above derivations, the dynamic, linear available power constraint (11) is reformulated to a system of linear constraints that is used for the quadratic programming problem of the MPO (9).

3) Dynamic Flow Predictor: The Dynamic Flow Predictor [8] is used to model the effect of wind turbine operation on the dynamics of wind speed at the wind turbines. The input to the Dynamic Flow Predictor is the power setpoints of the wind turbines. A dynamic flow model and a turbine model are used to calculate the outputs of the Dynamic Flow Predictor, i.e., turbine power output and the wind speed at wind turbines.

The flow model estimates wind turbine aerodynamic interaction using a delay process and a linearized engineering wake model, i.e., in this work, the Frandsen wake model [13]. The flow model is built as a dynamic, discrete-time state-space model as

$$
\begin{aligned}
\underbrace{\left[\begin{array}{c}
\vec{u}_{\text {del,all }} \\
\vec{u}_{0}
\end{array}\right]}_{\vec{x}}[n+1]= & \underbrace{\left[\begin{array}{cc}
\mathbf{A}_{1,1} & \mathbf{A}_{1,2} \\
0 & \mathbf{I}
\end{array}\right]}_{\mathbf{A}} \underbrace{\left[\begin{array}{c}
\vec{u}_{\text {del,all }} \\
\vec{u}_{0}
\end{array}\right]}_{\vec{x}}[n] \\
& +\underbrace{\left[\begin{array}{c}
\mathbf{B}_{\Delta P, \text { all }} \\
0
\end{array}\right]}_{\mathbf{B}} \underbrace{\Delta \vec{P}[n]}_{\vec{v}} \\
\vec{u}[n]= & \underbrace{\left[\begin{array}{cc}
\mathbf{C}_{u, 1,1} & 0
\end{array}\right]}_{\mathbf{C}_{u}} \underbrace{\left[\begin{array}{c}
\vec{u}_{\text {del, all }} \\
\vec{u}_{0}
\end{array}\right]}_{\vec{x}}[n]
\end{aligned}
$$

where $\vec{u}_{\text {del, all }}$ is the wind speed delay states of all wind turbines and $\vec{u}_{0}$ is the wind speed linearization point. The output of the flow model is the current rotor effective wind speed $\vec{u}$ at the turbines in the wind farm. $\Delta \vec{P}$ is the deviation of the turbine power setpoints from the power linearization point. The matrices $\mathbf{A}_{1,1}$ and $\mathbf{A}_{1,2}$ model the aerodynamic interaction of wind turbines. Matrix $\mathbf{C}_{u}$ relates the wind speed states $\vec{u}_{\text {del, all }}$ to the current rotor effective wind speed $\vec{u}$ at the turbines in the wind farm. The turbine model uses a direct feed-through to model power output, and as such the turbine power output is modeled as equal to the turbine power setpoint. To improve the prediction accuracy of the Dynamic Flow Predictor, a Kalman filter [14] and a dynamic system update are employed. Tests of the Dynamic Flow Predictor show that it can accurately capture the dynamics of wind speed modeled by a higher fidelity simulation model. More information on the Dynamic Flow Predictor model can be found in [8].

\section{LARGE-ScAle Wind FARM CASE StUdy}

The performance of the MPO-based dispatch is compared to the other dispatch functions in a simulation case study of a large-scale wind farm. The objective is to demonstrate the performance of the MPO-based dispatch in a demanding scenario. As such, the use of a large-scale wind farm results in larger computational costs, and increased complexity in the wind farm modeling and the MPO. In addition, the wind farm is requested to operate in delta-control mode [2], [15], which results in a demanding reference for the total power. More information on the delta-control mode can be found in Section III-A3.

The case study is structured into the simulation setup, i.e., the wind farm, the simulation environment, and the wind farm operation mode, and the simulation results, i.e., the performance of the Dynamic Flow Predictor and the dispatch functions, and the computation time of the MPO.

\section{A. Simulation Setup}

1) Wind Farm: The investigated, large-scale wind farm is generic, in order to avoid the association of the presented results with the operation of a particular, real wind farm. Nonetheless, the chosen layout and turbine model shall represent the operation of typical offshore wind farms [16]. Fig. 2 shows the layout of the wind farm. The spacing of the wind turbines is five rotor diameters between both rows and columns of the square-grid wind farm layout. The wind farm comprises 80 NREL 5-MW wind turbines [17] with a rotor diameter of $126 \mathrm{~m}$ and a rated wind speed of $11.4 \mathrm{~m} / \mathrm{s}$.

2) Simulation Environment: SimWindFarm: The investigated, large-scale wind farm is simulated using the dynamic simulation framework SimWindFarm [18]. SimWindFarm performs simultaneous, dynamic simulations of the wind turbines in the wind farm, the wind farm control, the aerodynamic interaction of the wind turbines and the actions by the transmission system operator. The NREL5MW virtual turbine model [17] is used to model wind turbine operation. The wind turbines are controlled using a standard wind turbine control approach [19]. The operation of the wind turbines is coordinated using the feedback controller presented in Section II-A, 


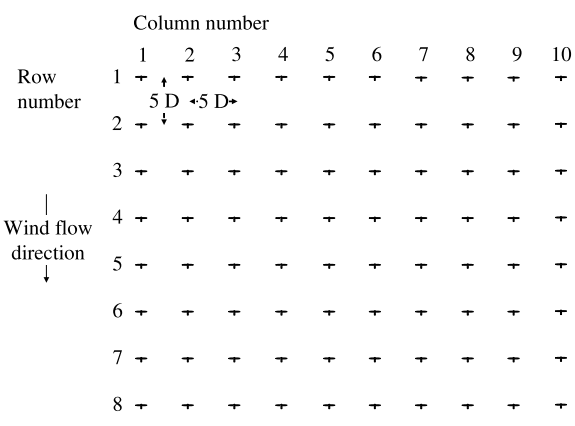

Fig. 2. Layout of simulated, generic 80 turbine wind farm used to compare dispatch functions of power controller.

which is embedded in the DTU Wind Farm Controller [20]. The DTU Wind Farm Controller is linked to the SimWindFarm simulation tool and replaces the basic, standard wind farm controller in SimWindFarm. In SimWindFarm, wind turbine aerodynamics is modeled using the turbine power coefficient and thrust coefficient. Up to third-order dynamic models are employed to simulate the drive train, generator, and pitch actuator. The aerodynamic model of the wind flow in the wind farm is structured into an ambient field model and a turbine wake model [13]. The ambient wind field is modeled as the hub height, turbulent wind flow advected with the mean wind speed under the assumption of Taylor's frozen turbulence. In [21], it is shown that in large-scale wind farms the power output of adjacent wind turbines can be strongly correlated with a coefficient of correlation of up to 0.8 out of 1 . Thus, in the conditions of this work, Taylor's hypothesis is considered a reasonable approximation. Wake flow modeling includes wake wind speed deficit, wake width expansion, wake meandering, and wake merging. More information on the aerodynamics of wakes in wind farms can be found in [16].

All simulations use the same wind conditions, i.e., a mean wind speed of $8 \mathrm{~m} / \mathrm{s}$ and a turbulence intensity of $6 \%$. The wind direction is aligned with the turbine rows as indicated in Fig. 2. The simulation duration is $5000 \mathrm{~s}$, while the discussion of simulation results focuses on the operation from $1000 \mathrm{~s}$ onward. In the first $1000 \mathrm{~s}$, the wake flow is building up, and thus, this time span is not considered in the analysis.

3) Wind Farm Operation Mode: In order to provide secondary regulation services, the wind farm is operated in the delta-control mode [2], [15], i.e., downregulation of the total wind farm power by a certain percentage. Therefore, the wind farm can provide secondary regulation by increasing the farm power output from the downregulated power level to the maximum power output. The total power reference signal $P_{\text {ref }}(t)$, as shown in Fig. 3, is thus defined as

$$
P_{\text {ref }}(t)=[1-\gamma] P_{\text {avail,avg }}
$$

where $\gamma$ is the amount of derating and $P_{\text {avail,avg }}$ is the backward-averaged total available power prior to the initiation of the control at time $t$. The averaging durations used in this work are 1 and $5 \mathrm{~min}$. The longer averaging duration results in less high-frequency content in the reference signal, but a larger time shift of the reference signal into the future as compared to the available power. The derating $\gamma$ is set to $4 \%$.

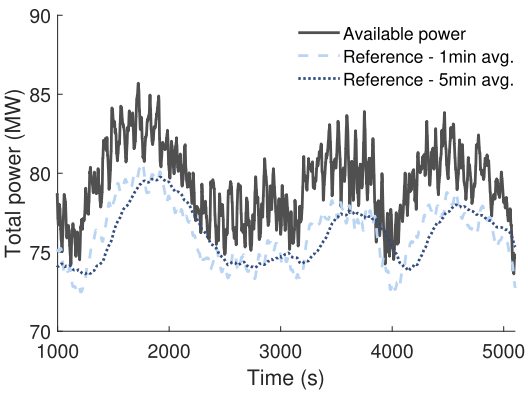

Fig. 3. Total wind farm power references used in case study on large-scale wind farm. Reference signals are set as $4 \%$ derating from 1 min (blue) and 5 min (yellow) average total available power of wind farm (gray) prior to initiation of control.

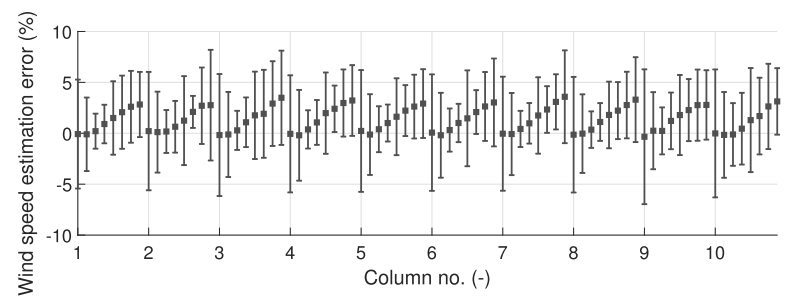

Fig. 4. Accuracy of wind speed estimates of Dynamic Flow Predictor in terms of mean, normalized error (square), and NRMS error (error bar). Reference is simulation environment SimWindFarm.

\section{B. Results}

The analysis of the case study results is focused on the accuracy of the Dynamic Flow Predictor, the effect of the dispatch function on the total power reference tracking, and the computation time of the MPO.

1) Dynamic Flow Predictor Performance: The performance of the MPO-based dispatch relies on the accuracy of the employed model, i.e., the Dynamic Flow Predictor. The accuracy of the Dynamic Flow Predictor is, therefore, investigated during the operation of the large-scale wind farm with the MPO-based dispatch, in the same conditions as used in the comparison with the other dispatch functions. Fig. 4 shows the mean and normalized root-mean-square (NRMS) error of wind speed estimates. The error is the difference between the estimate by the Dynamic Flow Predictor and SimWindFarm. It can be observed that the mean error increases with downstream turbines from no error to a maximum of $3.6 \%$ from the most upstream turbine to the most downstream turbine. It can be observed that the trend is the same for all turbine rows. The positive bias in the wind speed estimate results in an overestimation of the available turbine power. Since the estimate of available power is used as a constraint in the MPO-based dispatch, the derived power set-points are more likely to exceed the available power at further downstream turbines. The NRMS error of wind speed estimates increases with downstream turbines from $1.6 \%$ to a maximum of $5.4 \%$. The persistence-based prediction at upstream turbines ranges between $5.4 \%$ and $6.6 \%$. The model-based estimate at downstream turbines is, thus, more accurate than the persistence-based estimate at upstream turbines. More details on this phenomenon can be found in [8]. 


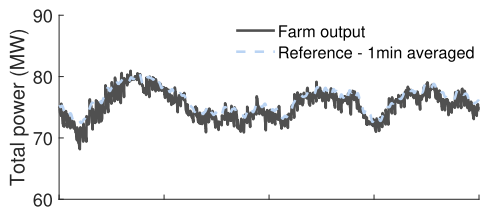

(a)

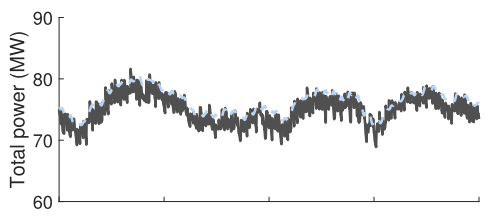

(c)

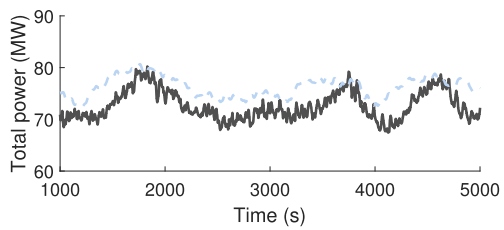

(e)

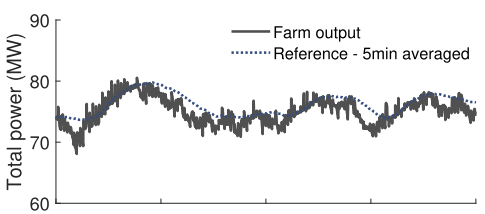

(b)

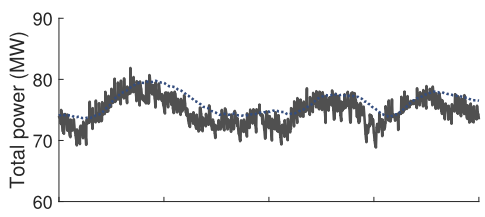

(d)

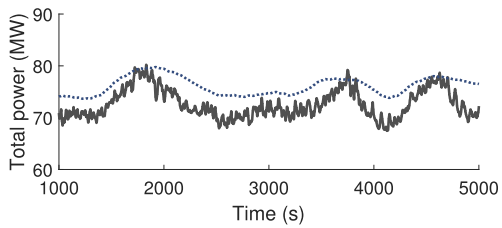

(f)

Fig. 5. Effect of dispatch function on tracking of total power reference in simulation of 80-turbine wind farm. Wind farm controller distributes demanded total power using (a) and (b) MPO-based dispatch function, (c) and (d) proportional dispatch function, and (e) and (f) static dispatch function. The reference signal is obtained based on 1-min averaged total available power (left) or 5-min averaged total available power (right).

2) Performance of MPO: The use of the MPO-based dispatch is observed to improve the reference track during the operation in delta-control mode. The approach could, therefore, be used to enhance the quality of secondary regulation services.

The effect of the dispatch function on the tracking of the total power reference of the delta-control mode is discussed in more detail in the following. Fig. 5 shows the effect of the dispatch function on the time-resolved tracking of the total power reference. Overall, it can be observed that the tracking performance is the most accurate with the MPO-based dispatch, and the static dispatch performs worst. Fig. 5(a) and (b) shows the time series of the total power of the wind farm for operation with the MPO-based dispatch function. It can be observed that the reference is followed well, overall, and the farm power output tends to be below the reference signal. The use of the reference signal based on the 5-min averaged available power results in a worse tracking performance as compared to the reference based on the 1-min averaged available power. The worse tracking performance is observed in periods when the reference is decreasing, such as, for example, from 2000 to $2500 \mathrm{~s}$. In such periods, the larger, negative phase shift in the reference signal introduced by the longer, 5-min backward-averaging of the available power results in a more demanding reference signal, as can be seen in Fig. 3.

Fig. 5(c) and (d) shows the time series of the total power of the wind farm for operation with the proportional dispatch function. The reference tracking behavior is similar to the operation with the MPO-based dispatch function. However, a larger variation of the total power and a lower mean total

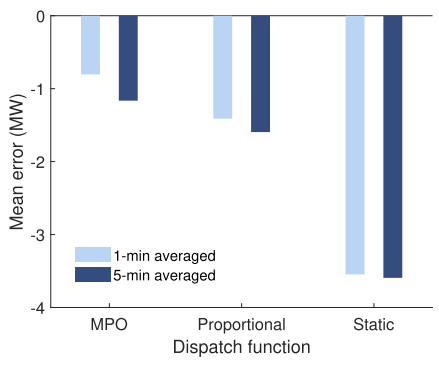

Fig. 6. Effect of dispatch function on mean error from total power reference in simulation of 80 turbine wind farm.

power is observed. Fig. 5(e) and (f) shows the time series of the total power of the wind farm for operation with the static dispatch function. It can be observed that the tracking is worse than with the other two dispatch functions. With the static dispatch, the wind farm is only able to deliver the power requested by the reference at few time instances. This is because the demanded total power is distributed equally to the wind turbines, and thus some wind turbines are requested to produce more power than their available power. As a result, there is a steady state error from the total power reference as discussed in Section II-A3 on controller tuning.

The tracking error from the total power reference is quantified in Figs. 6 and 7 in terms of the mean error and the NRMS error, respectively. It can be observed that operation with the reference based on shorter averaging of 1 min reduces the errors of the MPO-based dispatch and proportional dispatch by $30 \%$ and $14 \%$, respectively. There is no effect observed for the static dispatch since the total power reference is 


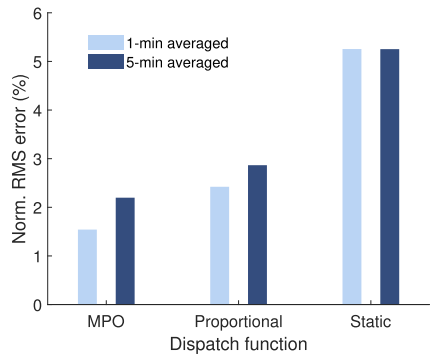

Fig. 7. Effect of dispatch function on NRMS error of total power reference in simulation of 80 turbine wind farm.

above the farm power output independent of the averaging duration used for the generation of the reference signal. The results, therefore, show that a shorter averaging duration, employed for the generation of the reference signal used in the delta-control mode, can result in a lower reference following error. The following discussion, thus, focuses on the results for the reference based on $1 \mathrm{~min}$ average. The lowest error is observed with the MPO dispatch function, which shows a mean error of $-0.8 \mathrm{MW}$ and an NRMS error of $1.5 \%$. The reduction of the mean error with respect to the proportional dispatch and the static dispatch is $43 \%$ and $77 \%$, respectively. The reduction of the NRMS error with respect to the proportional dispatch and the static dispatch is $36 \%$ and $71 \%$, respectively.

The improvement in the tracking of the reference for the total power of the wind farm is mainly due to three reasons. First, the Dynamic Flow Predictor provides a more accurate prediction of the available power at wind turbines than the persistence-based prediction used in the proportional dispatch. The performance improvement achieved with the MPO dispatch is likely to be lower in the real wind farm, because of a lower accuracy of the Dynamic Flow Predictor. On the other hand, advances of the Dynamic Flow Predictor such as the automatic correction of the mean prediction error could improve the performance of the MPO dispatch further. Second and third, in the tuning of the MPO dispatch, it was observed that the use of a prediction horizon and the penalization of turbine power variation increases the power tracking accuracy.

3) MPO Computation Time: The computation time of the MPO is two orders of magnitude faster than comparable model predictive controllers in the literature. Smaller computation time is beneficial as it results in a reduced delay in applying the optimized turbine power distribution to the wind turbines. The computation time of the MPO dispatch is on average $0.68 \mathrm{~s}$, which is composed of $0.27 \mathrm{~s}$ for the optimization and $0.41 \mathrm{~s}$ for the model update. The computation time is calculated on a standard personal computer for the current MATLAB implementation of the MPO dispatch. Optimizing the implementation in terms of employed hardware and software can further reduce the computation time. In [7], a comparable, however, nonlinear model-predictive controller is applied to an 80-turbine wind farm for active power control. The computation time of the nonlinear optimization is quantified as $60 \mathrm{~s}$, i.e., two orders of magnitude larger than the computation time of this work.

\section{CONCLUSION}

The brief proposes an optimization-based dispatch function for power control of wind farms during ancillary services. The commonly employed proportional dispatch is simple and robust, but only considers the availability of aerodynamic power at wind turbines for distributing the total, requested power to the wind turbines. The use of an optimization-based dispatch function allows deciding on the distribution of total power based on multiple objectives, models of the wind farm operation and possible, future trajectories of operation. The proposed optimization-based dispatch function uses model-predictive, multi-objective optimization. The optimization objectives are to follow a reference for the total power of the wind farm and to penalize variations of turbine power. The employed model is the Dynamic Flow Predictor, which uses a Kalman filter-driven feedback to correct the wind farm flow model dynamically.

The developed, optimization-based dispatch function is compared in dynamic simulation to dispatch functions commonly employed in present wind farms in a secondary regulation scenario. The comparison is carried out on an 80-turbine, large-scale wind farm. This work, hence, shows that the developed dispatch function is scalable to large wind farms. The newly developed dispatch function yields a reduction of the mean error and the normalized-root-meansquare error of $43 \%$ and $36 \%$ with respect to the proportional dispatch function. Another achievement is the speed of the optimization of only $0.21 \mathrm{~s}$ for a large-scale wind farm, i.e., two orders of magnitude faster than comparable approaches in the literature. Response time within $1 \mathrm{~s}$ provides enough bandwidth to execute other required services of secondary power system regulation like voltage and frequency control.

The future work aims at extending the objectives of the optimization-based dispatch to include the mitigation of turbine fatigue loads. Next, the enhancement of the Dynamic Flow Predictor could further improve the performance of the optimization-based dispatch. Third, it is of interest to compare the use of linear and nonlinear models in the optimization-based dispatch.

\section{ACKNOWLEDGMENT}

The authors would like to thank Vattenfall $R \& D$ and Siemens Gamesa Renewable Energies for the fruitful discussions.

\section{REFERENCES}

[1] “Global wind report 2017," Global Wind Energy Council, Brussels, Belgium, Tech. Rep., 2017. [Online]. Available: https://gwec.net/costcompetitiveness-puts-wind-in-front/

[2] J. Kristoffersen, "The horns rev wind farm and the operational experience with the wind farm main controller," Proc. Copenhagen Offshore Wind, vol. 122, no. 2, pp. 1-26, 2005.

[3] J.-W. van Wingerden, L. Pao, J. Aho, and P. Fleming, "Active power control of waked wind farms," IFAC-PapersOnLine, vol. 50, no. 1, pp. 4484-4491, Jul. 2017. 
[4] A. D. Hansen, P. Sørensen, F. Iov, and F. Blaabjerg, "Centralised power control of wind farm with doubly fed induction generators," Renew. Energy, vol. 31, no. 7, pp. 935-951, Jun. 2006.

[5] M. Soleimanzadeh, R. Wisniewski, and S. Kanev, "An optimization framework for load and power distribution in wind farms," J. Wind Eng. Ind. Aerodyn., vols. 107-108, pp. 256-262, 2012.

[6] J. Kazda, K. Merz, J. Tande, and N. Cutululis, "Mitigating turbine mechanical loads using engineering model predictive wind farm controller," J. Phys. Conf. Ser., vol. 1104, no. 1, 2018, Art. no. 012036.

[7] C. R. Shapiro, J. Meyers, C. Meneveau, and D. F. Gayme, "Wind farms providing secondary frequency regulation: Evaluating the performance of model-based receding horizon control," Wind Energy Sci., vol. 3, no. 1, pp. 11-24, Mar. 2018.

[8] J. Kazda and N. A. Cutululis, "Fast control-oriented dynamic linear model of wind farm flow and operation," Energies, vol. 11, no. 12, p. 3346, 2018.

[9] S. Boersma, B. Doekemeijer, M. Vali, J. c, and J.-W. V. Wingerden, "A control-oriented dynamic wind farm model: WFSim," Wind Energy Sci., vol. 3, no. 1, pp. 75-95, 2018.

[10] P. Fleming, J. Aho, P. Gebraad, L. Pao, and Y. Zhang, "Computational fluid dynamics simulation study of active power control in wind plants," in Proc. Amer. Control Conf., Jul. 2016, pp. 1413-1420.

[11] P. M. O. Gebraad, P. A. Fleming, and J. W. Van Wingerden, "Wind turbine wake estimation and control using FLORIDyn, a control-oriented dynamic wind plant model," in Proc. Amer. Control Conf., Jul. 2015, pp. 1702-1708.

[12] J. B. Rawlings, "Tutorial overview of model predictive control," IEEE Control Syst., vol. 20, no. 3, pp. 38-52, Jun. 2000.
[13] S. Frandsen, R. Barthelmie, S. Pryor, O. Rathmann, S. Larsen, and J. Hojstrup, "Analytical modelling of wind speed deficit in large offshore wind farms," Wind Energy, vol. 9, nos. 1-2, pp. 39-53, Apr. 2006.

[14] R. E. Kalman and R. S. Bucy, "New results in linear filtering and prediction theory," J. Basic Eng., vol. 83, no. 1, pp. 95-108, Mar. 1961.

[15] Energinet.dk. (2015). Technical Regulation 3.2.5 for Wind Power Plants with a Power Output Above $11 \mathrm{~kW}$. [Online]. Available: https://bit.ly/2uvuP57

[16] B. Sanderse, "Aerodynamics of wind turbine wakes: Literature review," Energy Res. Center Netherlands (ECN) Petten, The Netherlands, Tech. Rep. p. 46, 2009.

[17] J. Jonkman, S. Butterfield, W. Musial, and G. Scott, Definition of a 5-MW Reference Wind Turbine for Offshore System Development, document NREL/TP-500-38060, 2009.

[18] J. Grunnet, M. Soltani, and T. Knudsen, "Aeolus toolbox for dynamics wind farm model, simulation and control," in Proc. Eur. Wind Energy Conf., Aug. 2010, p. 10.

[19] L. Y. Pao and K. E. Johnson, "A tutorial on the dynamics and control of wind turbines and wind farms," in Proc. Amer. Control Conf., Jun. 2009, pp. 2076-2089.

[20] J. Kazda, T. Gogmen, G. Giebel, M. Courtney, and N. A. Cutululis, "Framework of multi-objective wind farm controller applicable to real wind farms," WindEurope Summit, Sep. 2016.

[21] S. J. Andersen, J. N. Sørensen, and R. F. Mikkelsen, "Turbulence and entrainment length scales in large wind farms," Phil. Trans. R. Soc. A, vol. 375 , no. 2091, 2017, Art. no. 20160107. 